\title{
Disproof of the Riemann Hypothesis
}

\author{
Frank Vega
}

CopSonic, 1471 Route de Saint-Nauphary 82000 Montauban, France

\begin{abstract}
Let's define $S(x)=\vartheta(x)-x$, where $\vartheta(x)$ is the Chebyshev function. We prove that the Riemann Hypothesis is false when $\int_{x}^{\infty} \frac{S(y) \times(1+\log y)}{y^{2} \times \log ^{2} y} d y \geq \frac{S(x)^{2}}{x^{2} \times \log x}$ is satisfied for some number $x \geq 121$. In addition, we demonstrate that the previous inequality is satisfied when $S(x) \geq 0$ for some number $x \geq 121$. It is known that $S(x)$ changes sign infinitely often. In this way, we show that the Riemann Hypothesis is indeed false.
\end{abstract}

Keywords: Riemann hypothesis, Chebyshev function, Nicolas inequality, Prime numbers 2000 MSC: 11M26, 11A41, 11A25

\section{Introduction}

In mathematics, the Riemann Hypothesis is a conjecture that the Riemann zeta function has its zeros only at the negative even integers and complex numbers with real part $\frac{1}{2}$ [1]. Let $N_{n}=$ $2 \times 3 \times 5 \times 7 \times 11 \times \cdots \times p_{n}$ denotes a primorial number of order $n$ such that $p_{n}$ is the $n^{\text {th }}$ prime number. Say Nicolas $\left(p_{n}\right)$ holds provided

$$
\prod_{q \mid N_{n}} \frac{q}{q-1}>e^{\gamma} \times \log \log N_{n} .
$$

The constant $\gamma \approx 0.57721$ is the Euler-Mascheroni constant, log is the natural logarithm, and $q \mid N_{n}$ means the prime number $q$ divides to $N_{n}$. The importance of this property is:

Theorem 1.1. [2], [3]. Nicolas $\left(p_{n}\right)$ holds for all prime numbers $p_{n}>2$ if and only if the Riemann Hypothesis is true.

In mathematics, the Chebyshev function $\vartheta(x)$ is given by

$$
\vartheta(x)=\sum_{p \leq x} \log p
$$

where $p \leq x$ means all the prime numbers $p$ that are less than or equal to $x$. We know this:

Theorem 1.2. [4].

$$
\lim _{x \rightarrow \infty} \frac{\vartheta(x)}{x}=1
$$

Email address: vega.frank@gmail.com (Frank Vega) 
Theorem 1.3. [5]. For $x>1$ :

$$
\vartheta(x)<\left(1+\frac{1}{2 \times \log x}\right) \times x .
$$

Let's define $S(x)=\vartheta(x)-x$. It is a known result that:

Theorem 1.4. [6]. S $(x)$ changes sign infinitely often.

We also know that

Theorem 1.5. [3]. For $x \geq 121$.

$$
\log \log \vartheta(x) \geq \log \log x+\frac{S(x)}{x \times \log x}-\frac{S(x)^{2}}{x^{2} \times \log x}
$$

Let's define $H=\gamma-B$ such that $B \approx 0.2614972128$ is the Meissel-Mertens constant [7]. We know from the constant $H$, the following formula:

Theorem 1.6. [8].

$$
\sum_{q}\left(\log \left(\frac{q}{q-1}\right)-\frac{1}{q}\right)=\gamma-B=H .
$$

For $x \geq 2$, the function $u(x)$ is defined as follows

$$
u(x)=\sum_{q>x}\left(\log \left(\frac{q}{q-1}\right)-\frac{1}{q}\right) .
$$

Nicolas showed that

Theorem 1.7. [3]. For $x \geq 2$ :

$$
0<u(x) \leq \frac{1}{2 \times(x-1)}
$$

Let's define:

$$
\delta(x)=\left(\sum_{q \leq x} \frac{1}{q}-\log \log x-B\right) .
$$

Robin theorem states the following result:

Theorem 1.8. [9]. $\delta(x)$ changes sign infinitely often.

In addition, the Mertens second theorem states that:

Theorem 1.9. [7].

$$
\lim _{x \rightarrow \infty} \delta(x)=0 .
$$

Besides, Rosser and Schoenfeld derived a remarkable identity:

Theorem 1.10. [5].

$$
\sum_{q \leq x} \frac{1}{q}=\log \log x+B+\frac{S(x)}{x \times \log x}-\int_{x}^{\infty} \frac{S(y) \times(1+\log y)}{y^{2} \times \log ^{2} y} d y .
$$


We define another function:

$$
\varpi(x)=\left(\sum_{q \leq x} \frac{1}{q}-\log \log \vartheta(x)-B\right) .
$$

Putting all together yields the proof that the inequality $\varpi(x)>u(x)$ is satisfied for a number $x \geq 3$ if and only if Nicolas $(p)$ holds, where $p$ is the greatest prime number such that $p \leq x$. In this way, we introduce another criterion for the Riemann Hypothesis based on the Nicolas criterion and deduce some of its consequences. One of these consequences is that the Riemann Hypothesis is indeed false.

\section{Results}

Theorem 2.1. The inequality $\varpi(x)>u(x)$ is satisfied for a number $x \geq 3$ if and only if $\operatorname{Nicolas}(p)$ holds, where $p$ is the greatest prime number such that $p \leq x$.

Proof. We start from the inequality:

$$
\varpi(x)>u(x)
$$

which is equivalent to

$$
\left(\sum_{q \leq x} \frac{1}{q}-\log \log \vartheta(x)-B\right)>\sum_{q>x}\left(\log \left(\frac{q}{q-1}\right)-\frac{1}{q}\right) .
$$

Let's add the following formula to the both sides of the inequality,

$$
\sum_{q \leq x}\left(\log \left(\frac{q}{q-1}\right)-\frac{1}{q}\right)
$$

and due to the theorem 1.6 , we obtain that

$$
\sum_{q \leq x} \log \left(\frac{q}{q-1}\right)-\log \log \vartheta(x)-B>H
$$

because of

$$
H=\sum_{q \leq x}\left(\log \left(\frac{q}{q-1}\right)-\frac{1}{q}\right)+\sum_{q>x}\left(\log \left(\frac{q}{q-1}\right)-\frac{1}{q}\right)
$$

and

$$
\sum_{q \leq x} \log \left(\frac{q}{q-1}\right)=\sum_{q \leq x} \frac{1}{q}+\sum_{q \leq x}\left(\log \left(\frac{q}{q-1}\right)-\frac{1}{q}\right) .
$$

Let's distribute it and remove $B$ from the both sides:

$$
\sum_{q \leq x} \log \left(\frac{q}{q-1}\right)>\gamma+\log \log \vartheta(x)
$$

since $H=\gamma-B$. If we apply the exponentiation to the both sides of the inequality, then we have that

$$
\prod_{q \leq x} \frac{q}{q-1}>e^{\gamma} \times \log \vartheta(x)
$$

which means that Nicolas $(p)$ holds, where $p$ is the greatest prime number such that $p \leq x$. The same happens in the reverse implication. 
Theorem 2.2. The Riemann Hypothesis is true if and only if the inequality $\varpi(x)>u(x)$ is satisfied for all numbers $x \geq 3$.

Proof. This is a direct consequence of theorems 1.1 and 2.1 .

Theorem 2.3. If the inequality $\varpi(x) \leq 0$ is satisfied for some number $x \geq 3$, then the Riemann Hypothesis should be false.

Proof. This is an implication of theorems 1.7, 2.1 and 2.2.

Theorem 2.4. If the inequalities $\delta(x) \leq 0$ and $S(x) \geq 0$ are satisfied for some number $x \geq 3$, then the Riemann Hypothesis should be false.

Proof. If the inequalities $\delta(x) \leq 0$ and $S(x) \geq 0$ are satisfied for some number $x \geq 3$, then we obtain that $\varpi(x) \leq 0$ is also satisfied, which means that the Riemann Hypothesis should be false according to the theorem 2.3 .

\section{Theorem 2.5.}

$$
\lim _{x \rightarrow \infty} \varpi(x)=0
$$

Proof. We know that $\lim _{x \rightarrow \infty} \varpi(x)=0$ for the limits $\lim _{x \rightarrow \infty} \delta(x)=0$ and $\lim _{x \rightarrow \infty} \frac{\vartheta(x)}{x}=1$. In this way, this is a consequence from the theorems 1.9 and 1.2.

Theorem 2.6. Under the assumption that

$$
\int_{x}^{\infty} \frac{S(y) \times(1+\log y)}{y^{2} \times \log ^{2} y} d y \geq \frac{S(x)^{2}}{x^{2} \times \log x}
$$

is satisfied for some number $x \geq 121$, then the Riemann Hypothesis should be false.

Proof. Under the assumption that

$$
\int_{x}^{\infty} \frac{S(y) \times(1+\log y)}{y^{2} \times \log ^{2} y} d y \geq \frac{S(x)^{2}}{x^{2} \times \log x}
$$

for some number $x \geq 121$, then we can deduce that

$$
\sum_{q \leq x} \frac{1}{q} \leq \log \log x+B+\frac{S(x)}{x \times \log x}-\frac{S(x)^{2}}{x^{2} \times \log x}
$$

according to the theorem 1.10 . Using the theorem 1.5 , then we obtain that

$$
\sum_{q \leq x} \frac{1}{q} \leq \log \log \vartheta(x)+B
$$

due to $x \geq 121$. However, that would mean

$$
\varpi(x)=\sum_{q \leq x} \frac{1}{q}-\log \log \vartheta(x)-B \leq 0
$$

and therefore, the Riemann Hypothesis should be false because of the theorem 2.3. 
Theorem 2.7. The inequality

$$
\int_{x}^{\infty} \frac{S(y) \times(1+\log y)}{y^{2} \times \log ^{2} y} d y \geq \frac{S(x)^{2}}{x^{2} \times \log x}
$$

is satisfied for some number $x \geq 121$ when $S(x) \geq 0$.

Proof. By the theorem 1.3, we have that:

$$
\begin{aligned}
\frac{S(x)}{x} & =\frac{\vartheta(x)-x}{x} \\
& <\frac{\left(1+\frac{1}{2 \times \log x}\right) \times x-x}{x} \\
& =\frac{x \times\left(\left(1+\frac{1}{2 \times \log x}\right)-1\right)}{x} \\
& =\left(1+\frac{1}{2 \times \log x}-1\right) \\
& =\frac{1}{2 \times \log x} \\
& <1
\end{aligned}
$$

for a number $x \geq 121$. In this way, we have that $0 \leq \frac{S(x)}{x}<1$ when $S(x) \geq 0$. Consequently, we obtain that $\frac{S(x)}{x} \geq \frac{S(x)^{2}}{x^{2}}$ under the assumption that $S(x) \geq 0$, since for every real number $0 \leq z<1$, the inequality $z \geq z^{2}$ is always satisfied. For that reason, we will have that $\frac{S(x)}{x \times \log x} \geq \frac{S(x)^{2}}{x^{2} \times \log x}$. However, we know that

$$
\int_{x}^{\infty} \frac{S(y) \times(1+\log y)}{y^{2} \times \log ^{2} y} d y=\frac{S(x)}{x \times \log x}+\epsilon
$$

such that $\epsilon$ could be a positive value which depends mostly on the number $x \geq 121$ and $S(x) \geq 0$. In conclusion, the theorem is true for some number $x \geq 121$ when $S(x) \geq 0$.

Theorem 2.8. The Riemann Hypothesis is indeed false.

Proof. This is a direct consequence of theorems 1.4, 2.6 and 2.7.

\section{References}

[1] P. B. Borwein, S. Choi, B. Rooney, A. Weirathmueller, The Riemann Hypothesis: A Resource for the Afficionado and Virtuoso Alike, Vol. 27, Springer Science \& Business Media, 2008.

[2] J.-L. Nicolas, Petites valeurs de la fonction d'Euler et hypothese de Riemann, Séminaire de Théorie des nombres DPP, Paris 82 (1981) 207-218.

[3] J.-L. Nicolas, Petites valeurs de la fonction d'Euler, Journal of number theory 17 (3) (1983) 375-388. doi:10.1016/0022-314X(83)90055-0.

[4] T. H. Grönwall, Some asymptotic expressions in the theory of numbers, Transactions of the American Mathematical Society 14 (1) (1913) 113-122. doi:10.2307/1988773.

[5] J. B. Rosser, L. Schoenfeld, Approximate Formulas for Some Functions of Prime Numbers, Illinois Journal of Mathematics 6 (1) (1962) 64-94. doi:10.1215/ijm/1255631807.

[6] D. J. Platt, T. S. Trudgian, On the first sign change of $\theta(x)-x$, Math. Comput. 85 (299) (2016) 1539-1547. doi:10.1090/mcom/3021. 
[7] F. Mertens, Ein Beitrag zur analytischen Zahlentheorie., J. reine angew. Math. 1874 (78) (1874) 46-62 doi:10.1515/crll.1874.78.46.

URL https://doi.org/10.1515/crll.1874.78.46

[8] Y. Choie, N. Lichiardopol, P. Moree, P. Solé, On Robin's criterion for the Riemann hypothesis, Journal de Théorie des Nombres de Bordeaux 19 (2) (2007) 357-372. doi:10.5802/jtnb.591.

[9] G. Robin, Sur l'ordre maximum de la fonction somme des diviseurs, Séminaire Delange-Pisot-Poitou Paris 82 (1981) 233-242. 